\title{
General Galilei Covariant Gaussian Maps
}

\author{
Giulio Gasbarri, ${ }^{1,2,3, *}$ Marko Toroš, ${ }^{2,3, \dagger}$ and Angelo Bassi ${ }^{2,3, \neq}$ \\ ${ }^{1}$ Abdus Salam ICTP, Strada Costiera 11, I-34151 Trieste, Italy \\ ${ }^{2}$ Department of Physics, University of Trieste, Strada Costiera 11, 34151 Trieste, Italy \\ ${ }^{3}$ Istituto Nazionale di Fisica Nucleare, Trieste Section, Via Valerio 2, 34127 Trieste, Italy
}

(Received 24 March 2017; published 8 September 2017)

\begin{abstract}
We characterize general non-Markovian Gaussian maps which are covariant under Galilean transformations. In particular, we consider translational and Galilean covariant maps and show that they reduce to the known Holevo result in the Markovian limit. We apply the results to discuss measures of macroscopicity based on classicalization maps, specifically addressing dissipation, Galilean covariance and non-Markovianity. We further suggest a possible generalization of the macroscopicity measure defined by Nimmrichter and Hornberger [Phys. Rev. Lett. 110, 16 (2013)].
\end{abstract}

DOI: 10.1103/PhysRevLett.119.100403

Introduction.-Symmetries have always played a central role in modern physics, especially after their mathematical formulation with the advent of group theory: they underlie the simplicity of nature and manifest the beauty of physical laws. They also serve as a guideline principle for deciding the form of the dynamics [1,2]. Here we are interested in the role of space-time symmetries in nonrelativistic quantum mechanics.

The covariance of the Schrödinger equation, and of the corresponding Liouville-von Neumann equation, under the action of the Galilean group, has been extensively discussed [3-5]. On the other hand, the investigation of Galilean covariance within the context of open quantum systems is still an area of active research [6]. The exact quantum dynamics of a system interacting with the surrounding environment can be very complicated: in general, heavy approximations and heuristical arguments are needed in order to arrive at an explicit useful expression for the system's effective dynamics. In this case, symmetries can be a guiding principle in constructing the effective dynamics, bypassing at least partially the complexity (or impossibility) of a direct calculation by imposing constraints, which are expected to hold not only at the fundamental level, but also at the effective level [7-15].

Space-time symmetries in open quantum systems have been fully analyzed only in the special, but very important, case of a Markovian, completely positive $(C P)$ and trace preserving (TP) dynamics. This dynamics, discussed in the seminal works of Gorini, Kossakowski, Sudarshan, and independently by Lindblad [16,17], is known as the quantum dynamical semigroup: it is generated by the Lindblad superoperator and can be written as a first order differential equation, called the Lindblad master equation. By imposing the additional request of covariance under the action of the Galilei group, Holevo in a series of works [18-21], completely characterized translational and Galilei covariant Lindblad master equations, by giving the explicit form of the Lindblad superoperators [22].
The Holevo characterizations play a major role in the description of several important physical phenomena such as environmental decoherence and relaxation phenomena [7-14]. Furthermore, they are also relevant for the foundations of quantum mechanics, where an intrinsic nonunitary dynamics is postulated to solve the measurement problem [23-25], the black hole information paradox [26], or to combine principles of general relativity with quantum mechanics [27].

Although the assumption of Markovianity is often well justified, recent technological advances have lead to investigating several phenomena exhibiting memory effects [28], e.g., ultrafast chemical reactions [29-34], side band cooling [35], and light harvesting in photosynthesis [36-41]. This is little surprising, as the time resolution of experimental apparata has increased severalfold in the last decades. It is therefore now clear that non-Markovian dynamics will acquire a more prominent role in the near future: the theoretical investigations are pressed by practical necessity.

In this Letter we will derive the general structure of nonMarkovian Galilei covariant Gaussian maps. More specifically, we will consider the non-Markovian Gaussian map introduced in Ref. [42], and we will impose covariance under Galilean space-time symmetries (translations, boosts and rotations). In this way we will obtain a generalization of the Holevo generators [18-21] to the non-Markovian Gaussian case. Using these results, we will discuss measures of macroscopicity based on classicalization maps. Specifically, we will address the role of non-Markovian and dissipative effects, which limit the validity of the macroscopicity measure proposed in Ref. [43].

General framework of Gaussian maps.-Non-Markovian dynamics are in general difficult to analyze: the system and environment form a complicated many-body problem which, without some additional simplifying assumption, remains intractable. On the other hand, the subclass of (non-Markovian) Gaussian maps, still appropriate for the 
description of a vast spectrum of phenomena [44-56], can be analyzed both analytically [42] and numerically [57,58].

The starting point of our analysis is the most general trace-preserving, completely positive Gaussian map derived in Ref. [42] (we work in interaction picture and adopt Einstein's summation convention):

$$
\begin{aligned}
\mathcal{M}_{t}= & \exp _{+}\left\{\int_{0}^{t} d \tau \int_{0}^{t} d s D_{j k}(\tau, s)\right. \\
& \left.\times\left(\hat{A}_{s L}^{k} \hat{A}_{\tau R}^{j}-\theta_{\tau s} \hat{A}_{\tau L}^{j} \hat{A}_{s L}^{k}-\theta_{s \tau} \hat{A}_{s R}^{k} \hat{A}_{\tau R}^{j}\right)\right\},
\end{aligned}
$$

where $\exp _{+}$denotes the time-ordered exponential, $D_{j k}$ is a complex valued positive semi-definite matrix, $\hat{A}$ are bounded Hermitian operators, and the subscript $L(R)$ denotes operators acting on the statistical operator $\rho$ from the left (right), e.g., $\hat{A}_{L}^{k} \hat{A}_{R}^{j} \hat{\rho}=\hat{A}^{k} \hat{\rho} \hat{A}^{j}$ with $\hat{A}^{k}$ Hermitian operators. The correlation matrix $D_{j k}(\tau, s)$ and the operators $\hat{A}^{k}$ are supposed to encode, phenomenologically, the action of the bath on the system. We note that, by imposing the request of Markovianity

$$
D_{j k}(\tau, s)=\delta(\tau-s) \tilde{D}_{j k}(s),
$$

where $\tilde{D}_{j k}(s)$ is a complex valued positive semi-definite matrix, the exponent in Eq. (1) takes the well-known Lindblad form.

Since we are interested in space-time symmetries, we now explicitly assume that the Hilbert space $\mathcal{H}_{\mathcal{S}}$ is $L^{2}\left(\mathbb{R}^{3}\right)$ (the generalization to the $N$-particle Hilbert space is straightforward). In this case it is convenient to decompose the operators in Eq. (1) by using the Weyl-Wigner decomposition (in Schrödinger picture) [59]:

$$
\hat{\mathbf{A}}_{t}=\int_{\mathbb{R}^{3}} d \alpha \int_{\mathbb{R}^{3}} d \boldsymbol{\beta} \mathcal{A}_{t}(\boldsymbol{\alpha}, \boldsymbol{\beta}) e^{i(\boldsymbol{\alpha} \cdot \hat{\mathbf{x}}+\boldsymbol{\beta} \cdot \hat{\mathbf{p}})},
$$

where $\hat{\mathbf{A}}_{t}$ may depend explicitly on time, which is encoded in the time-dependency of $\mathcal{A}_{t}$, and $\hat{\mathbf{x}}$ and $\hat{\mathbf{p}}$ are the standard position and momentum operators. It is then straightforward to show that the map in Eq. (1) becomes (in the interaction picture):

$$
\begin{aligned}
\mathcal{M}_{t}= & \exp _{+}\left\{\int d T \int d \Gamma \mathcal{D}\left(\boldsymbol{\alpha}_{1}, \boldsymbol{\beta}_{1}, \boldsymbol{\alpha}_{2}, \boldsymbol{\beta}_{2}, \tau, s\right) \Theta_{\tau s}^{\mu \nu}\right. \\
& \left.\times\left(e^{i\left(\boldsymbol{\alpha}_{1} \cdot \hat{\mathbf{x}}_{s \mu}+\boldsymbol{\beta}_{1} \cdot \hat{\mathbf{p}}_{\mu}\right)} e^{-i\left(\boldsymbol{\alpha}_{2} \cdot \hat{\mathbf{x}}_{\tau \nu}+\boldsymbol{\beta}_{2} \cdot \hat{\mathbf{p}}_{\nu}\right)}\right)\right\},
\end{aligned}
$$

where $d T=d \tau d s, d \Gamma=d \alpha_{1} d \boldsymbol{\beta}_{1} d \alpha_{2} d \boldsymbol{\beta}_{2}$, the integration domains, which we omit to simplify the notation, are $[0, t] \times[0, t]$ and $\otimes_{j=1}^{4} \mathbb{R}^{3}$ for the $T$ and $\Gamma$ integrals, respectively, $\hat{\mathbf{x}}_{s}$ is the position operator in the interaction picture at time $s, \mu$ and $\nu$ denote $L$ or $R$ (left or right operators), $\Theta_{\tau s}^{L R}=\Theta_{\tau s}^{R L}=1 / 2, \Theta_{\tau s}^{L L}=-\theta_{\tau s}, \Theta_{\tau s}^{R R}=-\theta_{s \tau}$ and

$\mathcal{D}\left(\boldsymbol{\alpha}_{1}, \boldsymbol{\beta}_{1}, \boldsymbol{\alpha}_{2}, \boldsymbol{\beta}_{2}, \tau, s\right)=D_{j k}(\tau, s) \mathcal{A}_{\tau}^{j}\left(\boldsymbol{\alpha}_{1}, \boldsymbol{\beta}_{1}\right) \mathcal{A}_{s}^{k}\left(\boldsymbol{\alpha}_{2}, \boldsymbol{\beta}_{2}\right)$

$$
\mathcal{D}\left(\boldsymbol{\alpha}_{1}, \boldsymbol{\beta}_{1}, \boldsymbol{\alpha}_{2}, \boldsymbol{\beta}_{2}, \tau, s\right)=\mathcal{D}^{*}\left(\boldsymbol{\alpha}_{2}, \boldsymbol{\beta}_{2}, \boldsymbol{\alpha}_{1}, \boldsymbol{\beta}_{1}, s, \tau\right) .
$$

We now impose the relevant Galilei symmetry on the system, constraining the form of the dynamics given by Eq. (4).

Covariance.-Let us consider a locally compact Lie group $G$ and a unitary representation $\hat{\mathbf{U}}_{g}$, with $g \in G$, on the Hilbert space of the system. Following [61,62] a quantum dynamical map is said to be $G$ covariant if it commutes with the linear transformation $\mathcal{U}_{g}[\cdot]=\hat{\mathbf{U}}_{g} \cdot \hat{\mathbf{U}}_{g}$ :

$$
\mathcal{M}_{t}=\mathcal{U}_{g}^{-1} \circ \mathcal{M}_{t} \circ \mathcal{U}_{g} .
$$

With reference to the single particle Hilbert space $\mathcal{H}_{\mathcal{S}}$ $\left(L^{2}\left(\mathbb{R}^{3}\right)\right)$ we assume that the Hamiltonian is covariant under the relevant symmetry of the Galilei group $\mathcal{G}$ [63]: specifically, we consider the centrally extended unitary representation $\left(\hat{\mathbf{U}}_{g}\right)$ of the Galilei group $(\mathcal{G})$ on $\mathcal{H}_{\mathcal{S}}$. The generators of infinitesimal translations, boosts, and rotations are (in the interaction picture)

$$
\begin{aligned}
\hat{\mathbf{p}} & =\hat{\mathbf{p}}, \\
\hat{\mathbf{J}} & =\hat{\mathbf{x}} \times \hat{\mathbf{p}}, \\
\hat{\mathbf{K}} & =m \hat{\mathbf{x}},
\end{aligned}
$$

respectively, where $m$ is the mass of the particle. Exploiting Eq. (4), and the fact that we are considering a unitary representation, it is straightforward to show that Eq. (7) is satisfied if and only if the following condition is satisfied:

$$
\begin{aligned}
& \int d T \int d \Gamma \mathcal{D}\left(\boldsymbol{\alpha}_{1}, \boldsymbol{\beta}_{1}, \boldsymbol{\alpha}_{2}, \boldsymbol{\beta}_{2}, \tau, s\right) \Theta_{\tau s}^{\mu \nu} \\
& \quad \times\left(e^{i\left(\boldsymbol{\alpha}_{1} \mathcal{U}_{g}\left[\hat{\mathbf{x}}_{s \mu}\right]+\boldsymbol{\beta}_{1} \mathcal{U}_{g}\left[\hat{\mathbf{p}}_{\mu}\right]\right)} e^{-i\left(\boldsymbol{\alpha}_{2} \mathcal{U}_{g}\left[\hat{\mathbf{x}}_{\tau \nu}\right]+\boldsymbol{\beta}_{2} \mathcal{U}_{g}\left[\hat{\mathbf{p}}_{v}\right]\right)}\right. \\
& \left.-e^{i\left(\boldsymbol{\alpha}_{1} \hat{\mathbf{x}}_{s \mu}+\boldsymbol{\beta}_{1} \hat{\mathbf{p}}_{\mu}\right)} e^{-i\left(\boldsymbol{\alpha}_{2} \hat{\mathbf{x}}_{\tau \nu}+\boldsymbol{\beta}_{2} \hat{\mathbf{p}}_{\nu}\right)}\right)=0 .
\end{aligned}
$$

This equation constrains the structure of the dynamical map under the Galilean symmetry $g \in \mathcal{G}$. In particular, we will now see how the request of translation (boost) covariance characterizes the structure of the dynamical map.

Translational covariance.-Restricting to the subgroup of translations $\mathcal{T} \subset \mathcal{G}$ we have that

$$
\begin{gathered}
\mathcal{U}_{a}\left[\hat{\mathbf{x}}_{t}\right]=\hat{\mathbf{x}}_{t}+\boldsymbol{a}, \\
\mathcal{U}_{\boldsymbol{a}}[\hat{\mathbf{p}}]=\hat{\mathbf{p}},
\end{gathered}
$$

where $\hat{\mathbf{x}}_{t}=\hat{\mathbf{x}}+(\hat{\mathbf{p}} / m) t$ is the position operator in the interaction picture at time $t, \boldsymbol{a}$ is a translation vector, and $\mathcal{U}_{\boldsymbol{a}}$ denotes the corresponding linear transformation [see Eq. (7)]. Using Eqs. (12), (13) we obtain from Eq. (11)

$$
\begin{aligned}
& \int d T \int d \Gamma \mathcal{D}\left(\boldsymbol{\alpha}_{1}, \boldsymbol{\beta}_{1}, \boldsymbol{\alpha}_{2}, \boldsymbol{\beta}_{2}, \tau, s\right) \Theta_{\tau s}^{\mu \nu} \\
& \quad \times e^{i\left(\boldsymbol{\alpha}_{1} \cdot \hat{\mathbf{x}}_{\mu s}+\boldsymbol{\beta}_{1} \cdot \hat{\mathbf{p}}_{\mu}\right)} e^{-i\left(\boldsymbol{\alpha}_{2} \cdot \hat{\mathbf{x}}_{\nu \tau}+\boldsymbol{\beta}_{2} \cdot \hat{\mathbf{p}}_{\nu}\right)}\left(1-e^{i\left(\boldsymbol{\alpha}_{1}-\boldsymbol{\alpha}_{2}\right) \cdot \boldsymbol{a}}\right)=0 .
\end{aligned}
$$

Since this relation must hold $\forall \boldsymbol{a}$, it follows that Eq. (14) is satisfied if and only if the following equality holds:

$\mathcal{D}\left(\boldsymbol{\alpha}_{1}, \boldsymbol{\alpha}_{2}, \boldsymbol{\beta}_{1}, \boldsymbol{\beta}_{2}, \tau, s\right)=\delta^{(3)}\left(\boldsymbol{\alpha}_{1}-\boldsymbol{\alpha}_{2}\right) \mathcal{D}_{T}\left(\boldsymbol{\alpha}_{1}, \boldsymbol{\alpha}_{2}, \boldsymbol{\beta}_{1}, \boldsymbol{\beta}_{2}, \tau, s\right)$, 
where $\mathcal{D}_{T}$ is a complex valued function, which we rewrite as $\mathcal{D}_{T}\left(\boldsymbol{\alpha}_{1}, \boldsymbol{\beta}_{1}, \boldsymbol{\alpha}_{2}, \boldsymbol{\beta}_{2}, \tau, s\right)=D_{j k}(\tau, s) \tilde{\mathcal{A}}_{\tau}^{j *}\left(\boldsymbol{\alpha}_{1}, \boldsymbol{\beta}_{1}\right) \tilde{\mathcal{A}}_{s}^{k}\left(\boldsymbol{\alpha}_{2}, \boldsymbol{\beta}_{2}\right)$.

We then insert Eq. (15) into Eq. (4), use Eq. (16), integrate over $\boldsymbol{\alpha}_{2}$ and relabel $\boldsymbol{\alpha}_{1}$ as $\boldsymbol{\alpha}$ to obtain

$$
\begin{aligned}
& \mathcal{M}_{t}=\exp _{+}\left\{\int_{0}^{t} d \tau \int_{0}^{t} d s \int_{\mathbb{R}^{3}} d \alpha D_{j k}(\tau, s)\right. \\
& \times\left(\left[F_{s L}^{k}(\hat{\mathbf{p}}, \boldsymbol{\alpha}) e^{i \alpha \cdot \hat{\mathbf{x}}_{s L}}\right]\left[F_{\tau R}^{j \dagger}(\hat{\mathbf{p}}, \boldsymbol{\alpha}) e^{-i \alpha \cdot \hat{\mathbf{x}}_{\tau R}}\right]\right. \\
& -\theta_{\tau S}\left[F_{\tau L}^{j \dagger}(\hat{\mathbf{p}}, \boldsymbol{\alpha}) e^{-i \alpha \cdot \hat{\mathbf{x}}_{\tau L}}\right]\left[e^{i \alpha \cdot \hat{\mathbf{x}}_{s L}} F_{s L}^{k}(\hat{\mathbf{p}}, \boldsymbol{\alpha})\right] \\
& \left.\left.-\theta_{s \tau}\left[F_{s R}^{k}(\hat{\mathbf{p}}, \boldsymbol{\alpha}) e^{i \alpha \cdot \hat{\mathbf{x}}_{s R}}\right]\left[e^{-i \alpha \cdot \hat{\mathbf{x}}_{\tau R}} F_{\tau R}^{k^{\dagger}}(\hat{\mathbf{p}}, \boldsymbol{\alpha})\right]\right)\right\},
\end{aligned}
$$

where

$$
F_{\tau \mu}^{k}(\hat{\mathbf{p}}, \boldsymbol{\alpha})=\int d \boldsymbol{\beta} \tilde{\mathcal{A}}_{\tau}^{k}(\boldsymbol{\alpha}, \boldsymbol{\beta}) e^{i \boldsymbol{\beta} \cdot \hat{\mathbf{p}}_{\mu}}
$$

is a completely general operator valued function of the operator $\hat{\mathbf{p}}$. Equation (17) fully characterizes translation covariant $C P$ Gaussian maps.

Boost covariance.-Restricting to the subgroup of boosts $\mathcal{B} \subset \mathcal{G}$ we have that

$$
\begin{gathered}
\mathcal{U}_{\boldsymbol{b}}\left[\hat{\mathbf{x}}_{t}\right]=\hat{\mathbf{x}}_{t}+t \boldsymbol{b} / m, \\
\mathcal{U}_{\boldsymbol{b}}[\hat{\mathbf{p}}]=\hat{\mathbf{p}}+\boldsymbol{b},
\end{gathered}
$$

where $\boldsymbol{b}=m \boldsymbol{v}$ is a momentum vector (a particle of mass $m$ boosted with velocity $v$ ) and $\mathcal{U}_{b}$ denotes the corresponding linear transformation [see Eq. (7)]. Imposing boost covariance, and following the analogous steps as for the characterization of translational covariance, we obtain the following equality:

$$
\begin{aligned}
\mathcal{D}\left(\boldsymbol{\alpha}_{1}, \boldsymbol{\alpha}_{2}, \boldsymbol{\beta}_{1}, \boldsymbol{\beta}_{2}, \tau, s\right)= & \delta^{(3)}\left(\boldsymbol{\beta}_{1}-\boldsymbol{\beta}_{2}+\boldsymbol{\alpha}_{1} \frac{s}{m}-\boldsymbol{\alpha}_{2} \frac{\tau}{m}\right) \\
& \times \mathcal{D}_{B}\left(\boldsymbol{\alpha}_{1}, \boldsymbol{\alpha}_{2}, \boldsymbol{\beta}_{1}, \boldsymbol{\beta}_{2}, \tau, s\right),
\end{aligned}
$$

where $\mathcal{D}_{B}$ is a complex valued function. Performing the following change of variables: $\boldsymbol{\beta}_{1} \rightarrow \boldsymbol{\beta}_{1}-\tau \alpha_{1} / m$ and $\boldsymbol{\beta}_{2} \rightarrow$ $\boldsymbol{\beta}_{1}-s \alpha_{2} / m$, using Eq. (21), we can then rewrite Eq. (4) as

$$
\begin{aligned}
& \mathcal{M}_{t}=\exp _{+}\left\{\int_{0}^{t} d \tau \int_{0}^{t} d s \int_{\mathbb{R}^{3}} d \boldsymbol{\beta} D_{j k}(\tau, s)\right. \\
& \times\left(\left[F_{s L}^{k}\left(\hat{\mathbf{x}}_{s}, \boldsymbol{\beta}\right) e^{i \boldsymbol{\beta} \cdot \hat{\mathbf{p}}_{L}}\right]\left[F_{\tau R}^{j \dagger}\left(\hat{\mathbf{x}}_{\tau}, \boldsymbol{\beta}\right) e^{-i \boldsymbol{\beta} \cdot \hat{\mathbf{p}}_{R}}\right]\right. \\
& -\theta_{\tau s}\left[F_{\tau L}^{j \dagger}\left(\hat{\mathbf{x}}_{\tau}, \boldsymbol{\beta}\right) e^{-i \boldsymbol{\beta} \cdot \hat{\mathbf{p}}_{L}}\right]\left[e^{i \boldsymbol{\beta} \cdot \hat{\mathbf{p}}_{L}} F_{s L}^{k}\left(\hat{\mathbf{x}}_{s}, \boldsymbol{\beta}\right)\right]
\end{aligned}
$$

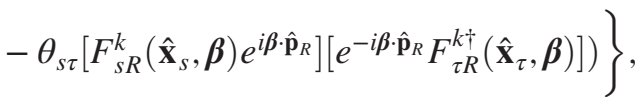

where

$$
F_{\tau \mu}^{k}\left(\hat{\mathbf{x}}_{\tau}, \boldsymbol{\beta}\right)=\int d \alpha \tilde{\mathcal{A}}_{\tau}^{k}(\boldsymbol{\alpha}, \boldsymbol{\beta}-\tau \alpha / m) e^{i \alpha \cdot \hat{\mathbf{x}}_{\tau \mu}}
$$

is a completely general operator valued function of the operator $\hat{\mathbf{x}}_{\tau}$. This equation completely characterizes boost covariant $C P$ Gaussian maps.

Translation-boost covariance.-We now require both translation and boost covariance. The dynamical map $\mathcal{M}_{t}$ must satisfy condition Eq. (15) as well as condition Eq. (21), i.e.,

$$
\begin{aligned}
\mathcal{D}\left(\boldsymbol{\alpha}_{1}, \boldsymbol{\alpha}_{2}, \boldsymbol{\beta}_{1}, \boldsymbol{\beta}_{2}, \tau, s\right)= & \delta^{(3)}\left(\boldsymbol{\alpha}_{1}-\boldsymbol{\alpha}_{2}\right) \\
& \times \delta^{(3)}\left(\boldsymbol{\beta}_{1}-\boldsymbol{\beta}_{2}+\boldsymbol{\alpha}_{1} \frac{s}{m}-\boldsymbol{\alpha}_{2} \frac{\tau}{m}\right) \\
& \times \mathcal{D}_{T B}\left(\boldsymbol{\alpha}_{1}, \boldsymbol{\alpha}_{2}, \boldsymbol{\beta}_{1}, \boldsymbol{\beta}_{2}, \tau, s\right) .
\end{aligned}
$$

Replacing Eq. (24) in Eq. (4), performing again the following change of variables: $\boldsymbol{\beta}_{1} \rightarrow \boldsymbol{\beta}_{1}-\tau \alpha_{1} / m$ and $\boldsymbol{\beta}_{2} \rightarrow \boldsymbol{\beta}_{1}-s \alpha_{2} / m$, one obtains

$$
\begin{aligned}
\mathcal{M}_{t}= & \exp _{+}\left\{\int_{0}^{t} d \tau \int_{0}^{t} d s \int d \alpha \int d \boldsymbol{F} \mathcal{F}(\boldsymbol{\alpha}, \boldsymbol{\beta}, \tau, s)\right. \\
& \times\left(e^{i\left(\boldsymbol{\alpha} \cdot \hat{\mathbf{x}}_{s L}+\boldsymbol{\beta} \cdot \hat{\mathbf{p}}_{L}\right)} e^{-i\left(\boldsymbol{\alpha} \cdot \hat{\mathbf{x}}_{\tau R}+\boldsymbol{\beta} \cdot \hat{\mathbf{p}}_{R}\right)}\right. \\
& -\theta_{\tau S} e^{-i\left(\boldsymbol{\alpha} \cdot \hat{\mathbf{x}}_{\tau L}+\boldsymbol{\beta} \cdot \hat{\mathbf{p}}_{L}\right)} e^{i\left(\boldsymbol{\alpha} \cdot \hat{\mathbf{x}}_{s L}+\boldsymbol{\beta} \cdot \hat{\mathbf{p}}_{L}\right)} \\
& \left.\left.-\theta_{s \tau} e^{-i\left(\boldsymbol{\alpha} \cdot \hat{\mathbf{x}}_{s R}+\boldsymbol{\beta} \cdot \hat{\mathbf{p}}_{R}\right)} e^{i\left(\boldsymbol{\alpha} \cdot \hat{\mathbf{x}}_{\tau R}+\boldsymbol{\beta} \cdot \hat{\mathbf{p}}_{R}\right)}\right)\right\}
\end{aligned}
$$

where $\mathcal{F}(\boldsymbol{\alpha}, \boldsymbol{\beta}, \tau, s)$ is a completely general real valued function. In this case the functional dependence of the map on the position and momentum operator is fixed [65]. This equation fully characterizes translation and boost covariant $C P$ Gaussian maps.

Rotation covariance.-For completeness, we also discuss rotation covariance. Restricting to the subgroup of rotations $\mathcal{R} \subset \mathcal{G}$ we have

$$
\begin{aligned}
\mathcal{U}_{R}\left[\hat{\mathbf{x}}_{s}\right] & =R \hat{\mathbf{x}}_{s}, \\
\mathcal{U}_{R}[\hat{\mathbf{p}}] & =R \hat{\mathbf{p}},
\end{aligned}
$$

where $R$ is a generic rotation matrix and $\mathcal{U}_{R}$ the corresponding linear transformation [see Eq. (7)]. Using the relation $\boldsymbol{a} \cdot(R \boldsymbol{b})=\left(R^{-1} \boldsymbol{a}\right) \cdot \boldsymbol{b}$, where $\boldsymbol{a}, \boldsymbol{b}$ are generic vectors, and recalling that the integral measure $d \alpha d \boldsymbol{\beta}$ is invariant under rotations, we perform the change of variables $\alpha \rightarrow R \alpha, \beta \rightarrow$ $R \boldsymbol{\beta}$ in Eq. (11), which gives the condition

$$
\mathcal{D}\left(R \alpha_{1}, R \boldsymbol{\beta}_{1}, R \alpha_{2}, R \boldsymbol{\beta}_{2}, \tau, s\right)=\mathcal{D}\left(\boldsymbol{\alpha}_{1}, \boldsymbol{\beta}_{1}, \boldsymbol{\alpha}_{2}, \boldsymbol{\beta}_{2}, \tau, s\right) .
$$

Equation (4), with the function $\mathcal{D}$ satisfying the symmetry given by Eq. (28), characterizes rotational covariant $C P$ Gaussian maps. This concludes the characterization of $C P$ Gaussian maps covariant under Galilean symmetries.

Markovian limits.-The $C P$ Gaussian covariant maps derived here above reduce to the well-known Markovian $C P$ Gaussian covariant maps in the Markovian limit. In particular, we immediately re-obtain the Holevo structures for the generators of the covariant quantum dynamical semigroup by imposing the request of Markovianity as given by 
Eq. (2). Under this assumption it is straightforward to show that Eq. (17) reduces to $\mathcal{M}_{t}=\exp _{+}\left\{\int_{0}^{t} d s \mathcal{L}_{s}\right\}$, where

$$
\begin{aligned}
\mathcal{L}_{s}= & \int d \alpha \tilde{D}_{j k}(s)\left(F_{s L}^{k}(\hat{\mathbf{p}}, \boldsymbol{\alpha}) e^{i \alpha \cdot \hat{\mathbf{x}}_{s L}} F_{s R}^{j \dagger}(\hat{\mathbf{p}}, \boldsymbol{\alpha}) e^{-i \alpha \cdot \hat{\mathbf{x}}_{s R}}\right. \\
& \left.-\frac{1}{2} F_{s L}^{j \dagger}(\hat{\mathbf{p}}, \boldsymbol{\alpha}) F_{s L}^{k}(\hat{\mathbf{p}}, \boldsymbol{\alpha})-\frac{1}{2} F_{s R}^{k}(\hat{\mathbf{p}}, \boldsymbol{\alpha}) F_{s R}^{k \dagger}(\hat{\mathbf{p}}, \boldsymbol{\alpha})\right),
\end{aligned}
$$

is the generator of the translational covariant semigroup.

Analogously, by considering the Markovian limit of the boost and translation covariant map derived in Eq. (25), we obtain the following generator:

$\mathcal{L}_{s}=\int d \alpha \int d \boldsymbol{\beta} \tilde{\mathcal{F}}(\boldsymbol{\alpha}, \boldsymbol{\beta}, s)\left(e^{i\left(\boldsymbol{\alpha} \cdot \hat{\mathbf{x}}_{s L}+\boldsymbol{\beta} \cdot \hat{\mathbf{p}}_{L}\right)} e^{-i\left(\boldsymbol{\alpha} \cdot \hat{\mathbf{x}}_{s R}+\boldsymbol{\beta} \cdot \hat{\mathbf{p}}_{R}\right)}-1\right)$,

where $\tilde{\mathcal{F}}$ is a positive valued function. Equations (29) and (30) correspond to the Holevo results for covariance under translation and boost-translation, respectively [20,21].

Macroscopicity measure.-More and more experiments are nowadays probing quantum mechanics in novel regimes, exploring in particular the boundary between quantum and classical [66-70]. It becomes relevant to define a measure that quantifies how far a given experiment pushes this boundary. This is a nontrivial task: what is the measure of macroscopicity that correctly accounts for complexity, size, mass, or some other feature of the system being explored?

Beginning with Leggett [71,72] several measures of macroscopicity have been proposed [43,73-78]. Among them, the one given by Nimmrichter and Hornberger in Ref. [78] has become quite popular in the matter-wave interferometry community because of its simplicity and versatility: they define as a macroscopicity measure a real number that quantifies how well an experiment tests a minimal modification of quantum mechanics. Specifically, they suggest the following measure:

$$
\mu=\log (\tau / 1 s)
$$

with $\tau$ the biggest excluded time scale in which quantum superpositions are suppressed by the minimal modification of quantum mechanics.

They further assume that the minimal modification of quantum mechanics, for a single particle with mass $m$, is described by a Markovian nonunitary TP, $C P$, Galilean covariant (translations, boosts and rotations) and time translation invariant map. This amounts to the nonunitary map generated by Eq. (30), where they choose the following parametrization of the correlation function:

$$
\tilde{\mathcal{F}}(\boldsymbol{\alpha}, \boldsymbol{\beta}, s)=\frac{1}{\tau} g(\boldsymbol{\alpha}, \boldsymbol{\beta}),
$$

where $g$ is a positive, isotropic phase-space distribution normalized to unity (a Gaussian function with variances $\sigma_{\alpha}$, $\sigma_{\beta}$ ) and $\tau$ gives the time scale in which superpositions are suppressed by the minimal modification (for further details see Refs. [43,78]).

The measure $\mu$ defined in Eq. (31) thus relies on the assumptions characterizing the minimal modification. Among these, Markovianity and Galilei covariance are usually taken for granted as they are a building block of the most successful nonrelativistic theories: quantum and classical mechanics. However, technological advances have come to the point of questioning the validity of these two assumptions; on top of this, minimal modifications need not satisfy them a priori. We take an example from the literature of collapse models, which can be seen as instances of minimal modifications of quantum mechanics in the spirit of Ref. [43]. $\mathrm{X}$-ray measurements [79] pose rather strong bounds on the collapse parameters [66]; however, the strength of the bounds depends critically on whether the collapse model is Markovian or not $[80,81]$. The reason is that such experiments explore the $\approx 10^{18} \mathrm{~Hz}$ region of the spectrum, meaning that the time resolution which is probed is $\approx 10^{-18} s$. Any cutoff in the spectrum of the collapse noise smaller than such frequencies weakens significantly the bound. A similar behavior is expected to occur for a macroscopicity measure that correctly includes non-Markovian effects. Markovianity might be verified only under a suitable temporal coarse graining of the underlying dynamics. In general if the time resolution of the experiment is longer than the correlation times associated to the modifications of the theory, then the Markovian assumption is justified, as any non-Markovian dynamics with finite correlation times may be approximated by its Markovian limit [82].

The assumption of Galilean covariance (translation and boost), even if it seems an innocent assumption, forces the nonunitary dynamics to produce an infinite growth of the system's energy on long time scales [85]. Galilean covariant maps must be then understood only as a good approximation that can be used in experiments that run for sufficiently short times, such that dissipative phenomena are negligible. In experiments with a long running time, the results could be influenced by dissipative phenomena and consequently the assumption of Galilean covariant dynamics is too restrictive. We consider a second example taken from collapse models. A recent experiment succeeded to cool a cloud of cold atoms to temperature less than $50_{-30}^{+50} \mathrm{pK}$ [87]. They measured the spreading of the cloud over time, which would be affected by modification of quantum mechanics. The analysis performed in Ref. [69] shows that the predictions of collapse models depend on whether dissipative effects are taken into account (Fig. (8) of Ref. [69] shows that the bounds on the collapse model drastically change with the thermalization temperature $T$, which quantifies the dissipation in the model). Again, a similar dependence on dissipation is expected by a macroscopicity measure, which takes dissipative effects into account.

To summarize, although $\mu$ is a reasonable choice for the measure of macroscopicity in many instances, novel 
experiments probing the very short and very long time scales need a different measure of macroscopicity due to NonMarkovian and dissipative effects, respectively. For such cases we propose to use the (translational covariant and nonMarkovian) map given in Eq. (17) as the minimal modification, with an appropriately chosen correlation function $D(t, s)$ and operators $F_{\tau \mu}(\hat{\mathbf{p}}, \boldsymbol{\alpha})$, where for simplicity we consider that the sum over $j, k$ contains only one term. We can still use Eq. (31) to define the measure of macroscopicity, where now $\tau \rightarrow \tau\left(\tau_{c}, T\right)$ is the biggest excluded time scale, for fixed parameters $\left(\tau_{c}, T\right)$, in which quantum superposition is suppressed by the minimal modification. Here $\tau_{c}$ is the correlation time of the correlation function $D(t, s)$ and $T$ is the temperature measuring dissipative effects.

To be more concrete we suggest the exponential correlation function

$$
D(t, s)=\frac{1}{2 \tau_{c}} e^{-|t-s| / \tau_{c}}
$$

and the Gaussian operators

$F_{\mu}(\hat{\mathbf{p}}, \boldsymbol{\alpha})=\sqrt{\frac{1}{\tau} \frac{m^{2}}{m_{0}^{2}}\left(\frac{r_{c}}{\sqrt{\pi} \hbar}\right)^{3}} e^{-\left(r_{c}^{2} / 2 \hbar^{2}\right)\left[\left(1+k_{T}\right) \boldsymbol{\alpha}+2 k_{T} \hat{\mathbf{p}}_{\mu}\right]^{2}}$,

where $k_{T}=\left(\hbar^{2} / 8 m_{0} r_{c}^{2} k_{B} T\right), m_{0}=1$ amu is a reference mass, $k_{B}$ is Boltzmann's constant, $r_{c}$ is a free length parameter analogous to the spread $\sigma_{\alpha}$ in Eq. (32), and $\tau$ gives the time scale in which the superpositions of a reference object with mass $\mathrm{m}$ are suppressed. In the Markovian $\left(\tau_{c} \rightarrow 0\right)$ and nondissipative $(T \rightarrow \infty)$ limit, we reobtain the measure of macroscopicity proposed by Nimmrichter and Hornberger with $\sigma_{\beta} \rightarrow 0$ [see Eqs. (31), (32)].

This new measure depends critically on the values of $\tau_{c}$ and $T$. To illustrate this, we have studied the classicalization map in the regime of small distances and low momentum transfer in one spatial dimension [88]. Specifically, we have considered a simple ideal experiment capable of resolving the time evolution of the spread of the wave packet of a freely evolving particle. The associated macroscopicity measure is investigated in the non-Markovian and dissipative regimes [89] (cf. Supplemental Material S4 and Fig. S2), showing how it depends on the correlation time $\tau_{c}$ and temperature $T$.

Summary.-We have analyzed Galilean symmetries in non-Markovian Gaussian $C P$ maps. The two main results of this Letter are the characterization of translational and of Galilei (translation-boost) covariant non-Markovian $C P$ Gaussian maps given by Eqs. (17) and (25), respectively. These maps are a generalization of the well-known Holevo results, which we reobtain in the Markovian limit. We have also provided the corresponding unravelling given by stochastic Schrödinger equations in a form suitable for nonperturbative numerical analysis [95]. As mentioned in the introduction, these results can find applications in several fields of research [7-14]. We have also analyzed the role that non-Markovian and dissipative effects play in the construction of a macroscopicity measure. We have shown that experiments probing the quantum-to-classical boundary on very short or very long time scales might not be adequately described by the macroscopicity measure in Ref. [43], and a more general definition is needed, as the one we propose, based on Eqs. (17), (33), and (34).

The authors acknowledge A. Smirne and A. Tilloy for insightful discussions. They also thank L. Ferialdi for insightful discussions and for the Mathematica codes of the cdQMUPL dynamics and gratefully acknowledge financial support from the University of Trieste (grant FRA 2016) and INFN. G. G. acknowledges financial support from ICTP Trieste.

G. G. and M. T. contributed equally to this work.

*giulio.gasbarri@ts.infn.it

marko.toros@ts.infn.it

tbassi@ts.infn.it

[1] R. P. Feynman, R. B. Leighton, and M. Sands, Quantum Mechanics The Feynman Lectures on Physics, Vol. III, Chap. 17 (Addison-Wesley, Reading, MA, 1966).

[2] M. E. Peskin, D. V. Schroeder, and E. Martinec, An Introduction to Quantum Field Theory (Addison-Wesley, Reading, MA, 1996).

[3] V. Bargmann, On unitary ray representations of continuous groups, Annals of Mathematics and Artificial Intelligence, 59, 1 (1954).

[4] L. E. Ballentine, Quantum Mechanics: A Modern Development (World Scientific, Singapore, 1998).

[5] S. T. Pereira and R. M. Angelo, Galilei covariance and Einstein's equivalence principle in quantum reference frames, Phys. Rev. A 91, 022107 (2015).

[6] H.-P. Breuer and F. Petruccione, The Theory of Open Quantum Systems (Oxford University Press on Demand, New York, 2002).

[7] B. Vacchini, Translation-covariant Markovian master equation for a test particle in a quantum fluid, J. Math. Phys. (N.Y.) 42, 4291 (2001).

[8] W. De Roeck and D. Spehner, Derivation of some translation-invariant Lindblad equations for a quantum Brownian particle, J. Stat. Phys. 150, 320 (2013).

[9] A. Isar, A. Săndulescu, and W. Scheid, Density matrix for the damped harmonic oscillator within the Lindblad theory, J. Math. Phys. (N.Y.) 34, 3887 (1993).

[10] Y. Yan, F. Shuang, R. Xu, J. Cheng, X.-Qi Li, C. Yang, and H. Zhang, Unified approach to the Bloch-Redfield theory and quantum Fokker-Planck equations, J. Chem. Phys. 113, 2068 (2000).

[11] A. Săndulescu and H. Scutaru, Open quantum systems and the damping of collective modes in deep inelastic collisions, Ann. Phys. (N.Y.) 173, 277 (1987).

[12] D. Kohen, C. Clay Marston, and D. J. Tannor, Phase space approach to theories of quantum dissipation, J. Chem. Phys. 107, 5236 (1997).

[13] B. Vacchini and K. Hornberger, Quantum linear Boltzmann equation, Phys. Rep. 478, 71 (2009). 
[14] D. I. Bondar, R. Cabrera, A. Campos, S. Mukamel, and H. A. Rabitz, Wigner-Lindblad equations for quantum friction, J. Phys. Chem. Lett. 7, 1632 (2016), PMID: 27078510.

[15] L. Ferialdi, Exact Closed Master Equation for Gaussian NonMarkovian Dynamics, Phys. Rev. Lett. 116, 120402 (2016).

[16] V. Gorini, A. Kossakowski, and E. C. G. Sudarshan, Completely positive dynamical semigroups of $n$-level systems, J. Math. Phys. (N.Y.) 17, 821 (1976).

[17] G. Lindblad, On the generators of quantum dynamical semigroups, Commun. Math. Phys. 48, 119 (1976).

[18] A. S. Holevo, A note on covariant dynamical semigroups, Rep. Math. Phys. 32, 211 (1993).

[19] A. S. Holevo, On conservativity of covariant dynamical semigroups, Rep. Math. Phys. 33, 95 (1993).

[20] A. S. Holevo, On translation-covariant quantum Markov equations, Izv. Math. 59, 427 (1995).

[21] A.S. Holevo, Covariant quantum Markovian evolutions, J. Math. Phys. (N.Y.) 37, 1812 (1996).

[22] The characterization is unique up to a unitary transformation (the same applies also for non-Markovian Gaussian maps discussed in this text).

[23] A. Bassi and G. Ghirardi, Dynamical reduction models, Phys. Rep. 379, 257 (2003).

[24] A. Bassi, K. Lochan, S. Satin, T. P. Singh, and H. Ulbricht, Models of wave-function collapse, underlying theories, and experimental tests, Rev. Mod. Phys. 85, 471 (2013).

[25] S. Weinberg, What happens in a measurement?, Phys. Rev. A 93, 032124 (2016).

[26] S. W. Hawking, Breakdown of predictability in gravitational collapse, Phys. Rev. D 14, 2460 (1976).

[27] R. Penrose, On gravity's role in quantum state reduction, Gen. Relativ. Gravit. 28, 581 (1996).

[28] H.-P. Breuer, E.-M. Laine, J. Piilo, and B. Vacchini. Colloquium: Non-Markovian dynamics in open quantum systems, Rev. Mod. Phys. 88, 021002 (2016).

[29] S. S. Plotkin and P. G. Wolynes, Non-Markovian Configurational Diffusion and Reaction Coordinates for Protein Folding, Phys. Rev. Lett. 80, 5015 (1998).

[30] A. I. Burshtein, Non-Markovian theories of transfer reactions in luminescence and chemiluminescence and photoand electrochemistry, Adv. Chem. Phys. 129, 105 (2004).

[31] A. Pomyalov and D. J. Tannor, The non-Markovian quantum master equation in the collective-mode representation: Application to barrier crossing in the intermediate friction regime, J. Chem. Phys. 123, 204111 (2005).

[32] E. Gindensperger, I. Burghardt, and L. S Cederbaum, Shorttime dynamics through conical intersections in macrosystems. I. Theory: Effective-mode formulation, J. Chem. Phys. 124, 144103 (2006).

[33] T. Guérin, O. Bénichou, and R. Voituriez, Non-Markovian polymer reaction kinetics, Nat. Chem. 4, 568 (2012).

[34] D. Chiarugi, M. Falaschi, D. Hermith, C. Olarte, and L. Torella, Modelling non-Markovian dynamics in biochemical reactions, BMC Systems Biology 9, 1 (2015).

[35] J. F. Triana, A. F. Estrada, and L. A. Pachón, Ultrafast Optimal Sideband Cooling under Non-Markovian Evolution, Phys. Rev. Lett. 116, 183602 (2016).

[36] L. A Pachón and P. Brumer, Computational methodologies and physical insights into electronic energy transfer in photosynthetic light-harvesting complexes, Phys. Chem. Chem. Phys. 14, 10094 (2012).
[37] L. A. Pachón and P. Brumer, Physical basis for long-lived electronic coherence in photosynthetic light-harvesting systems, J. Phys. Chem. Lett. 2, 2728 (2011).

[38] M. Schröder, U. Kleinekathöfer, and M. Schreiber, Calculation of absorption spectra for light-harvesting systems using non-Markovian approaches as well as modified redfield theory, J. Chem. Phys. 124, 084903 (2006).

[39] M. B. Plenio and S. F. Huelga, Dephasing-assisted transport: Quantum networks and biomolecules, New J. Phys. 10, 113019 (2008).

[40] M. Thorwart, J. Eckel, J. H. Reina, P. Nalbach, and S. Weiss, Enhanced quantum entanglement in the non-Markovian dynamics of biomolecular excitons, Chem. Phys. Lett. 478, 234 (2009).

[41] P. Nalbach, D. Braun, and M. Thorwart, Exciton transfer dynamics and quantumness of energy transfer in the fenna-matthews-olson complex, Phys. Rev. E 84, 041926 (2011).

[42] L. Diósi and L. Ferialdi, General Non-Markovian Structure of Gaussian Master and Stochastic Schrödinger Equations, Phys. Rev. Lett. 113, 200403 (2014).

[43] S. Nimmrichter and K. Hornberger, Macroscopicity of Mechanical Quantum Superposition States, Phys. Rev. Lett. 110, 160403 (2013).

[44] M. Tavis and F. W. Cummings, Exact solution for an $n$ molecule-radiation-field Hamiltonian, Phys. Rev. 170, 379 (1968).

[45] A. J. Leggett, S. Chakravarty, A. T. Dorsey, M. P. A. Fisher, A. Garg, and W. Zwerger, Dynamics of the dissipative twostate system, Rev. Mod. Phys. 59, 1 (1987).

[46] B. L. Hu, J. P. Paz, and Y. Zhang, Quantum Brownian motion in a general environment: Exact master equation with nonlocal dissipation and colored noise, Phys. Rev. D 45, 2843 (1992).

[47] L. Diósi, N. Gisin, and W. T. Strunz, Non-Markovian quantum state diffusion, Phys. Rev. A 58, 1699 (1998).

[48] A. D. Greentree, C. Tahan, J. H. Cole, and L. C. L. Hollenberg, Quantum phase transitions of light, Nat. Phys. 2, 856 (2006).

[49] S. L. Adler and A. Bassi, Collapse models with non-white noises. J. Phys. A 40, 15083 (2007).

[50] L. A. Pachon, G.-L. Ingold, and T. Dittrich, Nonclassical phase-space trajectories for the damped harmonic quantum oscillator, Chem. Phys. 375, 209 (2010).

[51] L. Diósi, Non-Markovian open quantum systems: Inputoutput fields, memory, and monitoring, Phys. Rev. A 85, 034101 (2012).

[52] C. Guo, A. Weichselbaum, J. von Delft, and M. Vojta, Critical and Strong-Coupling Phases in One-and Two-Bath Spin-Boson Models, Phys. Rev. Lett. 108, 160401 (2012).

[53] L. A. Pachón and P. Brumer, Mechanisms in environmentally assisted one-photon phase control, J. Chem. Phys. 139, 164123 (2013).

[54] C. Wang, J. Ren, and J. Cao, Nonequilibrium energy transfer at nanoscale: A unified theory from weak to strong coupling, Sci. Rep. 5, 11787 (2015).

[55] A. F. Estrada and L. A. Pachón, Quantum limit for driven linear non-Markovian open-quantum-systems, New J. Phys. 17, 033038 (2015).

[56] A. Tilloy, Interacting quantum field theories as relativistic statistical field theories of local beables, arXiv:1702.06325. 
[57] J. T. Stockburger and H. Grabert, Exact $c$-Number Representation of Non-Markovian Quantum Dissipation, Phys. Rev. Lett. 88, 170407 (2002).

[58] A. Tilloy, Markovian unraveling of non-Markovian stochastic Schrödingers equations, arXiv:1701.01948.

[59] H. Weyl, Quantenmechanik und gruppentheorie, Zeitschrift für Physik 46, 1 (1927).

[60] From the definition of $D_{j k}(\tau, s)$ in Ref. [42] we have $D_{k j}^{*}(s, \tau)=D_{j k}(\tau, s)$.

[61] E. Brian Davies, On the repeated measurement of continuous observables in quantum mechanics, J. Funct. Anal. 6, 318 (1970).

[62] A.S. Holevo, Statistical decision theory for quantum systems, J. Multivariate Anal. 3, 337 (1973).

[63] In the one particle case considered here it means that the Hamiltonian is $\hat{\mathbf{H}}=\hat{\mathbf{p}}^{2} / 2 \mathrm{~m}$. The many particle case, which is physically richer, as particle-particle interactions are allowed, can be treated in a similar way. See the discussion in Refs. [3-5] for a general discussion of the covariance of the dynamics (of isolated systems), as well as Ref. [64], for the covariance of the center of mass dynamics.

[64] L. L. Foldy, Relativistic particle systems with interaction, Phys. Rev. 122, 275 (1961).

[65] Clearly, Eq. (25) can be written in many different but equivalent ways by using unitary transformation on the operators.

[66] C. Curceanu, B. C. Hiesmayr, and K. Piscicchia, X-rays help to unfuzzy the concept of measurement, J. Adv. Phys. 4, 263 (2015).

[67] M. Toroš, G. Gasbarri, and A. Bassi, Bounds on collapse models from matter-wave interferometry, arXiv:1601.03672.

[68] M. Carlesso, A. Bassi, P. Falferi, and A. Vinante, Experimental bounds on collapse models from gravitational wave detectors, Phys. Rev. D 94, 124036 (2016).

[69] M. Bilardello, S. Donadi, A. Vinante, and A. Bassi, Bounds on collapse models from cold-atom experiments, Physica (Amsterdam) 462, 764 (2016).

[70] S. Belli, R. Bonsignori, G. D’Auria, L. Fant, M. Martini, S. Peirone, S. Donadi, and A. Bassi, Entangling macroscopic diamonds at room temperature: Bounds on the continuousspontaneous-localization parameters, Phys. Rev. A 94, 012108 (2016).

[71] A. J. Leggett, Macroscopic quantum systems and the quantum theory of measurement, Prog. Theor. Phys. Suppl. 69, 80 (1980).

[72] A. J. Leggett, Testing the limits of quantum mechanics: motivation, state of play, prospects, J. Phys. Condens. Matter 14, R415 (2002).

[73] W. Dür, C. Simon, and J. Ignacio Cirac, Effective Size of Certain Macroscopic Quantum Superpositions, Phys. Rev. Lett. 89, 210402 (2002).

[74] G. Björk and P. G. Luca Mana, A size criterion for macroscopic superposition states, J. Opt. B 6, 429 (2004).

[75] J. I. Korsbakken, K. Birgitta Whaley, J. Dubois, and J. Ignacio Cirac, Measurement-based measure of the size of macroscopic quantum superpositions, Phys. Rev. A 75, 042106 (2007).

[76] F. Marquardt, B. Abel, and J. von Delft, Measuring the size of a quantum superposition of many-body states, Phys. Rev. A 78, 012109 (2008).
[77] C.-W. Lee and H. Jeong, Quantification of Macroscopic Quantum Superpositions within Phase Space, Phys. Rev. Lett. 106, 220401 (2011).

[78] S. Nimmrichter, Macroscopic Matter Wave Interferometry (Springer, New York, 2014).

[79] C. E. Aalseth et al., Neutrinoless double- $\boldsymbol{\beta}$ decay of ${ }^{76} \mathrm{Ge}$ : First results from the international germanium experiment (IGEX) with six isotopically enriched detectors, Phys. Rev. C 59, 2108 (1999).

[80] S. L. Adler and F. M. Ramazanoglu, Photon-emission rate from atomic systems in the csl model, J. Phys. A 42, 109801 (2009).

[81] S. Donadi and A. Bassi, The emission of electromagnetic radiation from a quantum system interacting with an external noise: A general result, J. Phys. A 48, 035305 (2015).

[82] See Supplemental Material S1 at http://link.aps.org/ supplemental/10.1103/PhysRevLett.119.100403 for the discussion on the long time behavior of the statistical operator $\rho_{t}$ under the a generic Gaussian evolution in Eq. (1), which includes Refs. [83,84].

[83] L. Diósi, On high-temperature Markovian equation for quantum brownian motion, Europhys. Lett. 22, 1 (1993).

[84] A. O. Caldeira and A. J. Leggett, Path integral approach to quantum Brownian motion, Physica (Amsterdam) 121A, 587 (1983).

[85] See Supplemental Material S2 at http://link.aps.org/ supplemental/10.1103/PhysRevLett.119.100403 for a study on the asymptotic behavior of the energy for the GalileiCovariant dynamic in Eq. (4), which includes Refs. [18-21,86].

[86] A. Smirne and A. Bassi, Dissipative continuous spontaneous localization (csl) model, Sci. Rep. 5, 12518 (2015).

[87] T. Kovachy, J. M. Hogan, A. Sugarbaker, S. M. Dickerson, C. A. Donnelly, C. Overstreet, and M. A. Kasevich, Matter Wave Lensing to Picokelvin Temperatures, Phys. Rev. Lett. 114, 143004 (2015).

[88] We Taylor expand the operators in the time ordered exponential up to quadratic order in position and momentum operators, i.e., we keep only the terms proportional to $\hat{x}, \hat{p}, \hat{x}^{2}, \hat{x} \hat{p}, \hat{p} \hat{x}, \hat{p}^{2}$.

[89] See Supplemental Material S4 at http://link.aps.org/ supplemental/10.1103/PhysRevLett.119.100403 and Fig. S2 for the analysis of the macroscopicity measure in the nonMarkovian and dissipative regime, which includes Refs. [23,90-94]

[90] Lajos Diósi, Models for universal reduction of macroscopic quantum fluctuations, Phys. Rev. A 40, 1165 (1989).

[91] Lajos Diósi, Relativistic theory for continuous measurement of quantum fields, Phys. Rev. A 42, 5086 (1990).

[92] Luca Ferialdi and Angelo Bassi, Dissipative collapse models with nonwhite noises, Phys. Rev. A 86, 022108 (2012).

[93] Angelo Bassi and Luca Ferialdi, Non-Markovian Quantum Trajectories: An Exact Result, Phys. Rev. Lett. 103, 050403 (2009).

[94] Angelo Bassi, Emiliano Ippoliti, and Bassano Vacchini, On the energy increase in space-collapse models, J. Phys. A 38, 8017 (2005).

[95] See Supplemental Material S3 at http://link.aps.org/ supplemental/10.1103/PhysRevLett.119.100403 for the study on the stochastic unraveling that leads to $C P$ gaussian maps satisfying translation, boost, boost-translation, or rotation symmetry, which includes Refs. [41,57]. 